\title{
Trastornos de la conducta alimentaria en adolescentes: rol del pediatra. Recomendaciones de la Rama de Adolescencia de la Sociedad Chilena de Pediatría
}

\author{
Eating disorders in adolescents: role of the pediatrician. Recommendations \\ of the Adolescence Branch of the Chilean Society of Pediatrics
}

\author{
Verónica Gaete P., ${ }^{\mathrm{a}, \mathrm{b}}$, Carolina López C. ${ }^{\mathrm{a}, \mathrm{b}}$, Rama de Adolescencia Sociedad Chilena de Pediatría
}

${ }^{a}$ Centro SERJOVEN, Departamento de Pediatría y Cirugía Infantil Oriente, Facultad de Medicina, Universidad de Chile.

bUnidad de Trastornos Alimentarios, Centro de Adolescentes y Jóvenes, Departamento de Pediatría, Clínica Las Condes.

Recibido: 29 de noviembre de 2019; Aceptado: 20 de abril de 2020

¿Qué se sabe del tema que trata este estudio?

Los TCA son enfermedades muy serias que se inician habitualmente en la adolescencia. Existen intervenciones basadas en la evidencia para su prevención en la atención de adolescentes. Por otra parte, la pesquisa precoz y manejo adecuado de estas enfermedades resultan cruciales para un pronóstico favorable.

\section{¿Qué aporta este estudio a lo ya conocido?}

Recomendaciones prácticas de la Rama de Adolescencia de SOCHIPE sobre intervenciones que pueden realizarse durante la atención de adolescentes, para la prevención de los TCA, la pesquisa precoz y evaluación de quienes ya los presentan, y su derivación oportuna y eficaz a tratamiento especializado.

\section{Resumen}

Los trastornos de la conducta alimentaria (TCA) han adquirido relevancia en la pediatría chilena. $\mathrm{Su}$ tratamiento debe ser realizado, de preferencia, por equipos multidisciplinarios especializados o con alto grado de capacitación en la problemática. Sin embargo, los pediatras generales tienen un rol fundamental tanto en la prevención como en la pesquisa temprana de estas patologías. El objetivo de esta publicación es proporcionarles recomendaciones prácticas sobre las intervenciones que pueden llevar a cabo durante la atención de adolescentes, para la prevención de los TCA, la pesquisa precoz y evaluación de quienes ya los presentan, y su derivación oportuna a tratamiento especializado.
Palabras clave:

Trastornos alimentarios; adolescente; pediatría; prevención; detección temprana

Correspondencia:

Verónica Gaete P.

mgaete@med.uchile.cl 


\begin{abstract}
Eating disorders (ED) have become relevant in Chilean pediatrics. Their treatment must be preferably carried out by multidisciplinary teams with specialty or a high degree of training in the problem. However, general pediatricians have a fundamental role both in the prevention and in the early detection of these pathologies. The purpose of this publication is to provide them with practical recommendations on interventions that can be carried out during adolescent care for the prevention of ED, the early detection and evaluation of those who already have them, and their timely referral to specialized treatment.
\end{abstract}

Keywords:

Eating disorders; adolescent; pediatrics; prevention; early detection

\section{Introducción}

Los trastornos de la conducta alimentaria (TCA) han ido adquiriendo una progresiva relevancia en la pediatría chilena, pues se inician habitualmente en la adolescencia ${ }^{1}$, se asocian a significativa morbilidad y mortalidad $^{2,3} \mathrm{y}$, aunque debido a la escasez de estudios ello no se ha objetivado, existe en el ámbito clínico la percepción de que han aumentado significativamente.

Su tratamiento debe ser integral y realizado, de preferencia, por equipos multidisciplinarios especializados -o con alto grado de capacitación- tanto en TCA como en adolescencia ${ }^{4}$. Parte de los profesionales provendrá de subespecialidades de la Pediatría, en especial la Adolescencia y la Nutrición, además de otras especialidades necesarias para el manejo de las complicaciones médicas que estos casos presentan (por ej., endocrinología, ginecología, cardiología y gastroenterología).

Sin embargo, los pediatras generales están llamados a desempeñar un rol fundamental en el enfrentamiento de los TCA, pues por su amplia y estrecha relación con la población infanto-juvenil se encuentran en una posición privilegiada para favorecer su prevención y tratamiento temprano, el que es clave para un pronóstico favorable. El objetivo de esta publicación es proporcionarles recomendaciones prácticas sobre las intervenciones que pueden llevar a cabo durante la atención de adolescentes, para la prevención de los TCA, la pesquisa precoz y evaluación de quienes ya los presentan, y su derivación oportuna y eficaz a tratamiento especializado.

\section{Rol del pediatra}

\section{Prevención}

Los pediatras pueden contribuir a la prevención de los TCA implementando una serie de recomendaciones basadas en evidencia durante la atención de adolescentes. Éstas se abordan en un reporte clínico publicado en 2016 por los comités de Nutrición y Adolescencia, y la Sección de Obesidad de la Academia Americana de Pediatría ${ }^{1}$, quienes realizan las siguientes sugerencias para la prevención conjunta de los TCA y la obesidad:

a. Desaliente las dietas, el saltarse comidas y el uso de medicamentos para adelgazar; en cambio, fomente y apoye la implementación de conductas de alimentación y actividad física saludables que puedan mantenerse. El foco debe ponerse en tener una vida y hábitos saludables más que en el peso.

b. Promueva una imagen corporal positiva entre los adolescentes. No fomente la insatisfacción corporal ni se centre en ella como razón para hacer dieta. Se debe tener especial cuidado en no aumentar la insatisfacción corporal durante la atención de adolescentes con malnutrición por exceso, pues es habitual que ya la presenten, lo que incrementa su vulnerabilidad. Hay que ser delicado con los comentarios sobre su peso y/o figura, evitando utilizar palabras que puedan menoscabar su autoestima, tales como "gordo", "obeso" y "extremadamente obeso"s.

c. Estimule a las familias a que coman juntas con mayor frecuencia.

d. Aliéntelas a no conversar sobre el peso, sino más bien sobre alimentación y actividad física saludables para mantenerse sanos, $y$ a que faciliten ambas en sus hogares.

Desgraciadamente, el "weight/fat talk" (hablar, burlarse, etc., acerca del peso propio o de los demás) es frecuente hoy en día, con consecuencias negativas para la salud psicológica y física de los adolescentes, especialmente para las mujeres y si se da en el entorno familiar. El "fat talk" se asocia a TCA y a varios factores de riesgo de estas patologías, incluyendo -entre otros- insatisfacción corporal, preocupación por la imagen corporal, depresión, percepción de presión sociocultural para ser delgado e impulso hacia la delgadez ${ }^{6}$. Así también, el "weight-talk" de parte de los padres hacia sus hijos (incluso aquel bien intencionado, con la finalidad de estimular la baja de peso en aquellos con malnutrición por exceso), se asocia a una autopercepción corporal más pobre y un mayor involucramiento en dietas y conductas alimentarias disfuncionales ${ }^{7}$. 
e. Indague sobre antecedentes de maltrato o matonaje escolar en adolescentes con sobrepeso u obesidad, y aborde el problema con ellos y sus familias.

f. Monitoree cuidadosamente la baja ponderal en un adolescente que necesita perder peso, para asegurarse de que no desarrolle complicaciones médicas de la desnutrición.

\section{Pesquisa precoz}

La tendencia de quienes sufren los TCA a negarlos o esconderlos favorece su diagnóstico tardío, lo que impacta negativamente en sus posibilidades de recuperación ${ }^{8,9}$. Por ello, es importante que los pediatras los pesquisen activamente durante los controles de salud en la adolescencia ${ }^{10,11}$ y en la atención de aquellos jóvenes de riesgo ${ }^{12}$.

a. Screening de TCA en el control de salud del adolescente. Comprenderá la exploración del nivel de satisfacción del joven con su imagen corporal y las prácticas que utiliza para el control del peso, los que ayudarán a reconocer síntomas tempranos de $\mathrm{TCA}^{10}$. Asimismo, la historia menstrual -en el caso de las mujeres- y el examen físico podrán aportar información complementaria relevante. Este último, a través de la evaluación nutricional y del crecimiento y desarrollo del adolescente, mediante determinación de su peso, talla, IMC y estadios de Tanner, los que debiesen monitorizarse longitudinalmente.

b. Screening de TCA en la atención de adolescentes de riesgo. Debe realizarse de manera periódica ${ }^{12}$. Dentro de los factores de riesgo de TCA más factibles de ser pesquisados en la consulta, se encuentran: sexo femenino; antecedente familiar de TCA; pubertad temprana; insatisfacción con la imagen corporal y/o preocupación excesiva por la alimentación, el peso y la figura, con deseo de ser más delgado o de reducir alguna parte del cuerpo o aumentar la musculatura en el caso de muchos varones; internalización del "ideal delgado"; obesidad; hacer dietas; "weight talk" o "fat talk" parental; presión para ser delgado de parte de los padres y/o amigos; burlas por el peso de parte de familiares y/o amigos; práctica de ballet o deportes que exigen un cuerpo delgado; autoestima baja; perfeccionismo y depresión ${ }^{7,13-18}$. Los jóvenes con enfermedades crónicas que requieren un manejo nutricional específico (por ej., diabetes mellitus tipo 1, fibrosis quística, enfermedad celíaca y enfermedades inflamatorias intestinales) pueden tener también mayor riesgo de desarrollar un $\mathrm{TCA}^{12,19}$. Especial atención merecen además aquellos adolescentes cuyas consultas están relacionadas con el peso, la figura y/o alimentación, pues tienen asimismo un riesgo significativamente mayor de presentar estos trastornos ${ }^{20,21}$. c. Screening de TCA mediante instrumentos. Para simplificar el proceso de tamizaje de estas patologías y ponerlo al alcance de aquellos que no son especialistas, se han desarrollado instrumentos breves, que son fáciles y rápidos de administrar e interpretar. El SCOFF ${ }^{22}$ es el más estudiado y difundido de ellos. Sin embargo, la mayor parte de los estudios de validación de este instrumento han sido realizados en población adulta ${ }^{23}$, existiendo escasas investigaciones en adolescentes, por lo que su valor en este grupo etario está aún por determinarse.

En todos los casos previos, ante la sospecha de un TCA el pediatra deberá realizar una evaluación más profunda, como la que se sugiere a continuación.

\section{Evaluación inicial de los TCA}

Está destinada a efectuar el diagnóstico del TCA (y su tipo), detectar las complicaciones médicas asociadas, establecer la existencia de condiciones (médicas, psicológicas y sociales) que determinen la necesidad de hospitalización o intervenciones de urgencia, e informar al paciente y sus padres de los hallazgos y el tratamiento requerido, motivándolos a realizarlo. Para llevarla a cabo, deben tenerse en consideración las estrategias y habilidades básicas de atención clínica de adolescentes y las especificidades propias del abordaje de los TCA. Sobre las primeras se profundiza en un artículo publicado previamente por una de las autoras ${ }^{24}$. Las segundas se describen a continuación.

Es importante mantener una actitud de escucha, acogida y empatía durante la evaluación, mostrando a la vez una seriedad y preocupación acordes al problema, las que facilitarán que aquellos adolescentes y/o padres que no tengan conciencia de la relevancia estas enfermedades, la vayan desarrollando.

Debe destacarse que el diagnóstico de los TCA es eminentemente clínico y se realiza en especial mediante una buena anamnesis.

\section{Anamnesis}

Hay que explorar la existencia de alteraciones cognitivas y conductuales propias de los TCA, síntomas físicos asociados, antecedentes personales y familiares relevantes, tratamientos previos, y aquellos aspectos psicosociales que requieren de un abordaje urgente, además de evaluar el grado de comprensión del problema y la motivación a recibir ayuda tanto del paciente como de sus padres.

Alteraciones cognitivas y conductuales propias de los TCA

Para indagarlas, se recomiendan las siguientes preguntas $^{9,25,26}$, a las que se han añadido otras que se estiman útiles y necesarias: 
- ¿Cómo te sientes acerca de tu cuerpo? Si tuvieras una varita mágica y pudieras cambiar algo de él, ¿qué cambiarías? ¿Alguna vez has sentido miedo de aumentar de peso o volverte gordo?

- ¿ ¿Has tratado de bajar de peso? ¿Qué has intentado? ¿Desde cuándo?

- ¿Has disminuido el tamaño de las porciones que comes? ¿Te saltas comidas? ¿Qué alimentos que antes solías comer evitas o te prohíbes? ¿Desde cuándo presentas los previos? Explorar también rituales alimentarios y conversión reciente al vegetarianismo para el control del peso.

- ¿QQué comiste ayer? (cantidad y calidad del desayuno, almuerzo, onces, cena y colaciones).

- ¿Cuentas calorías? Si lo haces, ¿cuántas comes al día?

- ¿Tomas líquidos sin calorías (agua, té, café, gaseosas u otros) para evitar comer o bajar de peso? ¿Cuánto tomas al día? ¿Desde cuándo o en qué período?

- ¿Has tenido atracones? ¿Con qué frecuencia? ¿En qué horario? ¿Desde cuándo o en qué período? ¿Desencadenantes? ¿Restringes tu ingesta tras los atracones?

- ¿Te has provocado vómitos? ¿Con qué frecuencia? ¿En qué momento (después de un atracón o en otras circunstancias)? ¿Desde cuándo o en qué período?

- ¿Has tomado laxantes, diuréticos, medicamentos, productos "naturales" u otros para el control del peso? (en los varones, ¿has utilizado suplementos o medicamentos para aumentar tu masa muscular?) ¿De qué tipo, cuánto y con qué frecuencia? ¿En qué momento (después de un atracón o en otras circunstancias)? ¿Desde cuándo o en qué período?

- ¿Haces ejercicio? ¿De qué tipo y con qué intensidad, duración y frecuencia? ¿Cuánto te estresa perderte una sesión de ejercicio? ¿Lo has continuado realizando a pesar de estar enfermo o lesionado?

- ¿Con qué frecuencia te pesas?

- ¿Cuánto es lo que más has pesado? ¿Cuándo fue? ¿Cuánto medías en ese momento?

- ¿Cuál ha sido tu peso mínimo en el último año? ¿Cuándo fue? ¿Cuánto medías en ese momento?

- ¿Cuánto te gustaría pesar? ¿Cuál piensas que es tu peso saludable?

- ¿Miras, tocas o mides frecuentemente alguna(s) parte(s) de tu cuerpo con el fin de evaluar cómo está tu peso o figura?

- ¿Cuánto tiempo del día gastas pensando en la comida, el peso y la figura? ¿Cuánta de tu energía la inviertes en tu peso y tu figura?

Resulta importante explorar dirigidamente cada uno de estos aspectos, para que no pasen inadver- tidos. Se debe tener muy presente que la simple negación de problemas por parte del adolescente no excluye la posibilidad de que esté cursando con un TCA, y menos aún si sus padres, pares, profesores o entrenador lo sospechan, casos en los cuales la probabilidad de que el trastorno exista es alta ${ }^{27}$.

\section{Síntomas médicos asociados}

Se pueden revisar en el manuscrito "Trastornos de la conducta alimentaria en adolescentes. Una mirada integral" en este mismo número de la revista.

\section{Antecedentes personales y familiares relevantes}

Debe indagarse por el crecimiento y el desarrollo del adolescente, los antecedentes ginecológicos si es mujer (incluyendo edad de la menarquia, regularidad de los ciclos, fecha de la última regla y anticoncepción), y por historia familiar de obesidad, TCA y otros trastornos psiquiátricos ${ }^{25}$.

\section{Evaluación psicosocial}

Explorar la existencia de comorbilidad psiquiátrica que requiera de intervenciones de urgencia (por ejemplo, riesgo suicida) y conductas de riesgo que necesiten ser abordadas sin demora (por ejemplo, autoagresiones y relaciones sexuales no protegidas).

\section{Grado de comprensión del problema y motivación a recibir ayuda del paciente y sus padres}

Deben precisarse para realizar una buena derivación.

En general resulta muy relevante incluir a los padres en la anamnesis en los casos en que se sospecha un TCA, ya que frecuentemente la información que proviene de los pacientes es poco confiable. Sin embargo, hay que tener presente que a veces los padres no tienen conciencia del TCA o están en negación del problema. Se sugiere posponer el preguntar por cuestiones muy sensibles al adolescente para cuando se esté a solas con él, espacio que debe cautelarse con el propósito de respetar su progresiva autonomía y vincularse con él. No sólo las preguntas habitualmente sensibles (conducta sexual, consumo de drogas, etc.) lo son en estos casos, sino que también aquellas relativas a las conductas propias del TCA que los jóvenes desean mantener al margen del conocimiento de otros (por ej., vómitos y su frecuencia; atracones, su contenido y frecuencia; y uso de laxantes y otros medicamentos para el control del peso). La entrevista conjunta facilitará también que los padres tomen conciencia de lo que el adolescente ha vivenciado y permitirá evaluar las dinámicas asociadas a la enfermedad, entre ellas, el nivel de conflictos que el joven y su familia están teniendo durante las comidas, y cómo los están enfrentando. 
Otras habilidades que se deben tener especialmente presentes al realizar la anamnesis en estos casos son:

- Evitar juicios de valor y/o actitudes negativas o de sorpresa ante las conductas que al paciente ya le está siendo difícil compartir, pues se inhibirá de hacerlo y aumentarán sus resistencias a la situación.

- Externalizar la enfermedad. Esta técnica está indicada especialmente en pacientes resistentes a reconocer su problema y hacerse cargo de medidas para cambiar. En ella, se utiliza el lenguaje para convertir al TCA en una entidad separada del joven ("No eres tú, sino la anorexia la que hace que te comportes de esta forma"). Esto favorece que el adolescente pueda reconocer pensamientos y conductas que tiende a negar por culpa, vergüenza u otros motivos, y hace posible que el paciente "luche contra el TCA" (contra este "enemigo externo") en conjunto con su familia y el profesional, para impedir que "se salga con la suya". Permite desculpabilizar al paciente, sin que ello lo prive de la responsabilidad de superar el problema, lo que genera condiciones para que pueda contar lo que le está sucediendo y se movilice para solucionarlo.

\section{Examen físico}

Tiene que ser completo y debe incluir:

- Signos vitales: temperatura; frecuencia respiratoria; frecuencia cardíaca y presión arterial en decúbito dorsal y de pie.

- Antropometría: peso y talla.

- Cálculo del IMC y registro en curvas apropiadas ${ }^{28}$. El uso del IMC en estos casos puede ser insuficiente para realizar el diagnóstico nutricional, en especial en pacientes con Anorexia Nerviosa (AN) Atípica que iniciaron su TCA con malnutrición por exceso, pues a pesar de haber experimentado una pérdida de peso muy significativa y rápida, y presentar las mismas complicaciones que los casos de AN, pueden tener un IMC normal. La Sociedad de Salud y Medicina del Adolescente de EE. UU., basándose en las recomendaciones de la Academia
Americana de Pediatría, la Sociedad Americana de Nutrición Parenteral y Enteral, y la Academia de Nutrición y Dietética, propone clasificar el grado de desnutrición en pacientes con TCA tomando en consideración los parámetros incluidos en la tabla 1.

- $\quad$ Registro de talla en curvas apropiadas ${ }^{28}$, con observación de su evolución en relación con mediciones previas y realización del diagnóstico estatural.

- Desarrollo puberal: estadios de Tanner.

- Pesquisa de alteraciones asociadas a los TCA (ver en "trastornos de la conducta alimentaria en adolescentes. Una mirada integral" en este mismo número de la revista).

Se debe tener especial cuidado al momento de obtener el peso, por ser el objeto de gran parte de los temores de estos pacientes. El miedo a verse forzados a incrementarlo puede llevarlos a aumentarlo artificialmente mediante consumo excesivo de líquidos o escondiendo objetos en su ropa o cabello. Por lo mismo, el joven debe pesarse con un mínimo de ropa o en bata, y después de haber orinado. Posteriormente, se deben explorar sus sentimientos acerca del resultado, y, si está experimentando significativa frustración o angustia, será necesario contenerlo, empleando, por ejemplo, la externalización de la enfermedad ("comprendo que es la enfermedad la que te hace sentir que tu peso es excesivo, pero las curvas nos muestran que es absolutamente normal"). Si se anticipa que la reacción del adolescente al resultado del peso será demasiado intensa y entorpecerá la evolución, puede optarse porque no visualice la cifra.

\section{Diagnóstico diferencial (tabla 2)}

El de los TCA incluye esencialmente patologías médicas y psiquiátricas que se asocian a cambios significativos en el nivel de ingesta y/o peso corporal -disminución en el caso de la AN y aumento en el de la bulimia nerviosa $(\mathrm{BN})$ y trastorno por atracón-y/u obsesiones y comportamientos compulsivos respecto de la alimentación, el peso y/o la figura, además de vómitos si se trata de la $\mathrm{BN}^{29}$.

Tabla 1. Propuesta de clasificación del grado de desnutrición en adolescentes con Trastornos de Conducta Alimentaria

\begin{tabular}{llll}
\hline & Leve & Moderada & Severa \\
\hline \% mediana IMC & $80-90 \%$ & $70-79 \%$ & $<70 \%$ \\
puntaje z IMC & -1 a $-1,9$ & -2 a $-2,9$ & -3 o mayor \\
Pérdida de peso & $>10 \%$ de masa corporal & $>15 \%$ de masa corporal & $\begin{array}{l}>20 \% \text { de masa corporal en } 1 \text { año o }>10 \% \text { de masa } \\
\text { corporal en } 6 \text { meses }\end{array}$ \\
\hline
\end{tabular}

Uno o más de los previos podría sugerir desnutrición leve, moderada o severa. IMC: índice masa corporal. Golden et al., 2015³0; Society for Adolescent Health and Medicine, $2015^{33}$. 
Tabla 2. Diagnóstico diferencial de los Trastornos de Conducta Alimentaria

\begin{tabular}{ll}
\hline Anorexia nerviosa & Bulimia nerviosa o trastorno por atracón \\
\hline Patología endocrina: & Patología médica: \\
- Hipertiroidismo & - Obesidad \\
- Enfermedad de Addison & - Insulinoma \\
- Diabetes mellitus & - Tumores hipotalámicos \\
& - Obstrucción del vaciamiento gástrico \\
Patología gastrointestinal: & - Hiperemesis gravídica \\
- Enfermedad inflamatoria intestinal & - Síndrome de Prader-Willi \\
- Enfermedad celíaca & - Síndrome de Kleine-Levin \\
- Úlcera péptica & - Síndrone de Klüver-Bucy \\
Neoplasias: & Patología psiquiátrica: \\
- Tumores del sistema nervioso central & - Anorexia nerviosa (tipo con atracones/purgas) \\
- Otros cánceres & - Trastorno obsesivo-compulsivo \\
Otras patologías médicas: & - Trastornos depresivos \\
- Infecciones crónicas (por ej., tuberculosis) & Trastorno dismórfico corporal \\
Patología psiquiátrica: & \\
- Trastorno obsesivo-compulsivo & \\
- Trastornos ansiosos & \\
- Trastornos depresivos & \\
- Trastorno de evitación/restricción de la ingestión de alimentos & \\
\hline
\end{tabular}

Adaptado de Mairs y Nicholls, $2016^{29}$.

\section{Evaluación de laboratorio inicial ${ }^{25,30,31}$}

Los exámenes son solo complementarios y destinados a detectar complicaciones y descartar otras condiciones que puedan explicar los síntomas (diagnóstico diferencial). Deben incluir hemograma, perfil bioquímico, electrolitos plasmáticos, gases venosos, 25-hidroxi vitamina D y orina completa. También creatininemia, pruebas tiroideas y magnesemia, si existe baja de peso significativa. Si hay vómitos o sospecha de ellos se puede agregar una amilasemia. Se tiene que realizar un electrocardiograma (ECG) en todo paciente con alteraciones electrolíticas, baja de peso o purgas significativas, y/o síntomas o signos cardiovasculares, y considerar un ecocardiograma en los últimos. En aquellas adolescentes con amenorrea pueden indicarse exámenes adicionales (LH, FSH, estradiol), y, si se ha prolongado por 6 meses o más, debe llevarse a cabo una densitometría ósea, utilizando un programa computacional apropiado para la edad. En los varones con AN, debe considerarse la obtención de testosterona. Si hay incertidumbre acerca del diagnóstico, será necesario realizar otros exámenes según el caso, como VHS, pruebas para descartar enfermedad celíaca, tomografía computarizada o resonancia magnética de cerebro, y estudios del sistema gastrointestinal alto o bajo.

Las alteraciones que pueden mostrar los exámenes iniciales se detallan en la tabla 3. Sin embargo, pueden resultar normales, incluso si existe una desnutrición significativa ${ }^{2}$. Es importante representarle a los pacientes, ojalá por anticipado, que la normalidad de los exámenes no indica ausencia del TCA o de gravedad, para evitar que intenten utilizarla como argumento para negar el problema y resistirse a tratamiento.

\section{Devolución de la hipótesis diagnóstica e indicaciones}

Una vez completada la evaluación, el pediatra tiene que realizar un resumen al joven, dándole a conocer los hallazgos, diagnóstico(s), riesgos e indicaciones preliminares, incluyendo la necesidad de derivación a un programa de tratamiento ambulatorio especializado o a hospitalización/internación, según sea el caso. Debe también brindarle la oportunidad de expresar sus preocupaciones y resolver sus dudas. Si existen conductas propias del TCA que haya ocultado a sus padres (por ej., vómitos), el profesional tendrá que plantearle la necesidad de abrirlas a éstos, negociando -de manera empática y respetuosa- la mejor forma de hacerlo. Resulta esencial que los padres estén en conocimiento de este tipo de conductas para que reciba el tratamiento necesario. Finalmente, el pediatra tiene que dar un espacio a los padres para abordar los mismos aspectos, cuidando de mantener en confidencialidad la información sensible que corresponda y no diga relación con el TCA. 
Tabla 3. Alteraciones potenciales en los exámenes de laboratorio iniciales en Trastornos de Conducta Alimentaria

\begin{tabular}{ll}
\hline Examen & Alteraciones \\
\hline Hemograma & Anemia, leucopenia, trombocitopenia \\
Perfil bioquímico & Glucosa: $\downarrow$ (desnutrición) \\
& Nitrógeno ureico: $\uparrow$ (deshidratación) \\
& Calcio: leve $\downarrow$ (desnutrición, a expensas del hueso) \\
& Fósforo: $\downarrow$ (desnutrición) \\
& Proteínas totales/albúmina: $\uparrow$ en desnutrición temprana a expensas de la masa muscular, $\downarrow$ más tarde \\
& Alanina-aminotransferasa (ALAT, SGPT) y aspartato-aminotransferasa (ASAT, SGOT): $\uparrow$ (desnutrición) \\
& Sodio: $\downarrow$ (intoxicación hídrica o laxantes) \\
Electrolitos plasmáticos & Potasio: $\downarrow$ (vómitos, laxantes, diuréticos) \\
& Cloro: $\downarrow$ (vómitos, laxantes), $\uparrow$ (laxantes) \\
Gases venosos & Bicarbonato: $\uparrow$ (vómitos), $\downarrow$ (laxantes) \\
Magnesemia & $\downarrow$ (desnutrición, laxantes) \\
Creatininemia & $\uparrow$ (deshidratación, insuficiencia renal), $\downarrow$ (disminución masa muscular) \\
Amilasemia & $\uparrow$ (vómitos, pancreatitis) \\
Pruebas tiroideas & T3 $\downarrow$, T4 normal o $\downarrow$, TSH normal o $\downarrow$ (síndrome del eutiroideo enfermo) \\
Gonadotrofinas y esteroides sexuales & LH , FSH, estradiol: $\downarrow$ en mujeres \\
& Testosterona: $\downarrow$ en varones \\
ECG & Bradicardia y otras arritmias, intervalo QTc prolongado, aumento de la dispersión del intervalo QT \\
Densitometría ósea & Densidad mineral ósea $\downarrow$ \\
\hline
\end{tabular}

Adaptado de AED, 2016². ECG: Electrocardiograma.

Tabla 4. Indicaciones de hospitalización/internación en adolescentes con Trastornos de Conducta Alimentaria

Una o más de las siguientes justifican hospitalización:

1. $\mathrm{IMC} \leq$ al $75 \%$ del promedio para la edad y sexo

2. Deshidratación

3. Alteraciones electrolíticas (hipokalemia, hiponatremia, hipofosfemia)

4. Alteraciones electrocardiográficas (por ej., QTc prolongado, bradicardia severa)

5. Inestabilidad fisiológica

- Bradicardia severa (frecuencia cardíaca $<50$ latidos/min durante el día; $<45$ latidos/minuto en la noche

- Hipotensión (<90/45 mmHg)

- Hipotermia ( $\mathrm{T}^{\mathrm{a}}$ corporal $\left.<35,6^{\circ} \mathrm{C}\right)$

- Aumento ortostático del pulso (> 20 latidos/min) o disminución de la presión arterial (> $20 \mathrm{~mm} \mathrm{Hg}$ sistólica, > $10 \mathrm{mmHg}$ diastólica)

6. Detención del crecimiento y desarrollo

7. Rechazo agudo a comer

8. Atracones y purgas incontrolables

9. Complicaciones médicas agudas de la desnutrición (por ej., síncope, convulsiones, insuficiencia cardíaca, pancreatitis)

10. Comorbilidad psiquiátrica/médica que prohíbe o limita un tratamiento ambulatorio apropiado (por ej., depresión severa, ideación suicida, trastorno obsesivo-compulsivo, diabetes mellitus tipo 1)

11. Fracaso de tratamiento ambulatorio

Golden et al., 2015³. IMC: índice masa corporal.

\section{Derivación oportuna y eficaz a tratamiento especializado}

El pediatra deberá determinar si el paciente se encuentra en condiciones de recibir un tratamiento ambulatorio (especializado) o si su nivel de riesgo físico o psicosocial hacen necesaria una hospitalización o internación psiquiátrica (tabla 4), y derivarlo a una u otra instancia.

La derivación puede resultar todo un desafío, en especial cuando no solo el joven sino también sus padres evidencian resistencias al diagnóstico o tratamiento. Una derivación adecuada generalmente requerirá que los padres (e idealmente el adolescente, aunque puede no ser factible) logren reconocer la existencia del TCA, comprender sus riesgos, y entender la relevancia de no retrasar el tratamiento, aspectos que deberán ser abordados por el pediatra, con un tono de seriedad y preocupación acorde con los riesgos implicados. Asimismo, el profesional tiene que intentar comprender si hay otros motivos tras las resistencias y abordarlos. Por ejemplo, si la culpa es lo que está inmovilizando a los padres, el desculpabilizarlos y situarlos como agentes esenciales para la recuperación de su hijo puede ser muy útil. En caso de que los padres atribuyan las conductas del adolescente a vanidad o voluntarismo, será relevante hacerles ver que está enfermo; lo mismo si están esperando que sea su hijo quien quiera tratarse. 
Cuando existe mucha evitación puede ser conveniente no insistir en "ponerle nombre" a la enfermedad (o sea, no nombrarla por su diagnóstico: por ej., AN), para no incrementar los temores del paciente y/o sus padres. El trabajo motivacional puede complementarse con las estrategias de entrevista motivacional descritas por Miller y Rollnick ${ }^{32}$. Debe tenerse muy presente que será la motivación de los padres -y no la del adolescentela más determinante del ingreso a tratamiento, por la dependencia normal que tienen los jóvenes de ellos en esta etapa de la vida.

Aquellos casos con compromiso nutricional, médico y psicológico leves podrían eventualmente ser manejados por el pediatra con el apoyo de nutrición y salud mental, teniendo presente que deberá derivarlos sin demora a tratamiento especializado si no evolucionan satisfactoriamente.

\section{Conclusiones}

El tratamiento de los adolescentes con TCA debe ser integral y realizado, de preferencia, por equipos multidisciplinarios especializados o con alto grado de capacitación en la problemática. Sin embargo, los pediatras generales están llamados a desempeñar un rol fundamental en su prevención, pesquisa precoz y derivación oportuna a manejo especializado. Pueden contribuir a la prevención de los TCA implementando una serie de recomendaciones basadas en evidencia durante la atención de adolescentes. Deben realizar una pesquisa precoz tanto en los controles de salud en la adolescencia, como durante la atención de aquellos jóvenes de riesgo. Ante la sospecha de un TCA tendrán que llevar a cabo una evaluación más profunda, destinada a confirmar el diagnóstico, detectar las complicaciones médicas asociadas, establecer la existencia de condiciones que determinen la necesidad de hospitalización o intervenciones de urgencia, y motivar al paciente y muy especialmente a sus padres a iniciar un tratamiento. Para realizar lo previo, tienen que poseer conocimientos y habilidades de atención de adolescentes y otros(as) propios(as) del abordaje de los TCA. Por último, deberán derivar al paciente de manera oportuna y eficaz a tratamiento ambulatorio especializado $\mathrm{u}$ hospitalización/internación psiquiátrica si su nivel de riesgo físico o psicosocial lo justifica. Los pediatras generales podrían eventualmente optar por manejar -con el apoyo de nutrición y salud mental- aquellos casos con compromiso nutricional, médico y psicológico leves, derivando sin demora a aquellos pacientes que no evolucionen de manera satisfactoria.

Nota en relación con la terminología: A lo largo de este artículo se utiliza el término "pediatra" para denominar a todos aquellos médicos que se dedican a la atención clínica de niños y adolescentes. Por otra parte, cuando se hace mención de "el" o "los" adolescente(s) o joven(es), se está aludiendo a personas de diversos sexos de estos grupos etarios, y bajo la denominación "padre o padres" se incluyen a la(s) madre(s) y a otros adultos que ejerzan el rol parental.

\section{Conflicto de intereses}

Los autores declaran no tener conflicto de intereses.

\section{Referencias}

1. Golden NH, Schneider M, Wood C, AAP Committee on Nutrition. Preventing obesity and eating disorders in adolescents. Pediatrics. 2016;138(3): e20161649.

2. Academy for Eating Disorders (AED) report 2016. Eating disorders. A guide to medical care. Critical points for early recognition and medical risk management in the care of individuals with eating disorders. $3^{\text {rd }}$ Edition. 2016.

3. Jagielska G, Kacperska I. Outcome, comorbidity and prognosis in anorexia nervosa. Psychiatr Pol. 2017;51(2): 205-18.

4. Gaete V, López C, Matamala M, Raimann X: Trastornos de la conducta alimentaria en adolescentes y jóvenes. En: Cornejo V, Cruchet S. Nutrición en el ciclo vital; Santiago, Chile: Editorial

Mediterráneo, 2013:165-92.

5. Puhl RM, Peterson JL, Luedicke J. Parental perceptions of weight terminology that providers use with youth. Pediatrics. 2011;128(4):e786-93.

6. Shannon A, Mills JS. Correlates, causes, and consequences of fat talk: A review. Body Image. 2015;15:158-72.

7. Gillison FB, Lorenc AB, Sleddens EF, Williams SL, Atkinson L. Can it be harmful for parents to talk to their child about their weight? A meta-analysis. Prev Med. 2016;93:135-46.

8. López C, Treasure J. Trastornos de la conducta alimentaria en adolescentes: descripción y manejo. Rev Med Clin Condes. 2011;22(1):85-97.

9. Rome E. Eating disorders in children and adolescents. Curr Probl Pediatr Adolesc Health Care. 2012;42:28-44.
10. American Academy of Pediatrics: Adolescence Visits (11 Through 21 Years). En: Hagan JF, Shaw JS, Duncan PM, eds. Bright Futures: guidelines for health supervision of infants, children, and adolescents. 4th ed.; IL, USA: Elk Grove Village, 2017:731-822.

11. Ministerio de Salud, Gobierno de Chile. Orientaciones técnicas para el control de salud integral de adolescentes. 2016. Disponible en: https://diprece. minsal.cl/wrdprss_minsal/wp-content/ uploads/2018/02/OT-CONTROL-DESALUD-INTEGRAL-FINAL.pdf, última visita 26-02-20.

12. Campbell K, Peebles R. Eating disorders in children and adolescents: state of the art review. Pediatrics. 2014;34:582-92.

13. Keel P, Forney K. Psychosocial risk factors for eating disorders. Int J Eat Disord. 2013;46(5):433-9. 
14. Portela de Santana ML, da Costa Ribeiro H, Mora M, Raich RM. La epidemiología y los factores de riesgo de los trastornos alimentarios en la adolescencia; una revisión. Nutr Hosp. 2012;27(2):391-401.

15. Klump KL. Puberty as a critical risk period for eating disorders: a review of human and animal studies. Horm Behav. 2013;64(2):399-410.

16. Arcelus J. Witcomb GL, Mitchell A. Prevalence of eating disorders amongst dancers: a systemic review and meta-analysis. Eur Eat Disord Rev. 2014;22(2):92-101.

17. Schaumberg K, Welch E, Breithaupt L, et al. The science behind the Academy for Eating Disorders' nine truths about eating disorders. Eur Eat Disord Rev. 2017;25(6):432-50.

18. Joy E, Kussman A, Nattiv A. 2016 update on eating disorders in athletes: A comprehensive narrative review with a focus on clinical assessment and management. Br J Sports Med. 2016;50(3):154-62.

19. Quick VM, Byrd-Bredbenner C, Neumark- Sztainer D. Chronic illness and disordered eating: a discussion of the literature. Adv Nutr. 2013;4:277-86.

20. Lask B, Bryant-Waugh R, Wright F, Campbell M, Willoughby K, Waller G.
Family physician consultation patterns indicate high risk for early-onset anorexia nervosa. Int J Eat Disord. 2005;38:269-72.

21. Cebolla A, Perpiñá C, Lurbe E, AlvarezPitti J, Botella C. Prevalencia del trastorno por atracón en una muestra clínica de obesos. An Pediatr (Barc.). 2012;77(2):98102.

22. Morgan JF, Reid F, Lacey JH. The SCOFF questionnaire: assessment of a new screening tool for eating disorders. BMJ. 1999;319(7223):1467-8.

23. Lichtenstein MB, Hemmingsen SD, Støving RK. Identification of eating disorders symptoms in Danish adolescents with the SCOFF questionnaire. Nord J Psychiatry. 2017;71(5):340-7.

24. Gaete V. Atención clínica del adolescente que consulta por un problema de salud. Rev Med Clin Condes. 2011;22(1):5-13.

25. Rosen D. Identification and management of eating disorders in children and adolescents. Pediatrics. 2010;126:1240-53.

26. Sacco B, Kelley U. Diagnosis and evaluation of eating disorders in the pediatric patient. Pediatr Ann. 2018;47(6):e244-9.

27. Rome ES, Strandjord SE. Eating disorders. Pediatr Rev. 2016;37(8):323-36.

28. Ministerio de Salud, Gobierno de Chile. Norma para la evaluación nutricional de niños, niñas y adolescentes de 5 años a 19 años de edad. 2016. [Accedido 18.04.20] disponible en https://www.previenesalud. cl/assets/PDF/normas/2016-normaevaluacion-nutricional.pdf

29. Mairs R, Nicholls D. Assessment and treatment of eating disorders in children and adolescents. Arch Dis Child, 2016;101(12):1168-75.

30. Golden NH, Katzman DK, Sawyer SM, et al. Update on the medical management of eating disorders in adolescents. J Adolesc Health 2015;56(4):370-5.

31. Academy for Eating Disorders (AED) report 2011. Eating disorders. Critical points for early recognition and medical risk management in the care of individuals with eating disorders. $2^{\text {nd }}$ Edition. 2011.

32. Miller W, Rollnick S. Motivational interviewing: Helping people change. $3^{\text {rd }}$ ed.; New York, USA: The Guilford Press, 2013.

33. Golden NH, Katzman DK, Sawyer SM, et al; Society for Adolescent Health and Medicine. Position paper of the Society for Adolescent Health and Medicine: medical management of restrictive eating disorders in adolescents and young adults. J Adolesc Health 2015;56(1):121-5. 